\title{
Stability of stratified spin-up flows
}

\author{
S. A. Smirnov \\ Mechanical Engineering Department, Texas Tech University, USA
}

\begin{abstract}
The stability of stratified rotating flows is investigated by means of laboratory experiments in axisymmetric cylindrical and annular containers with both horizontal and sloping bottoms. The baroclinic current is initiated via incremental spin-up/down of a linearly stratified fluid by an abrupt change in the rotation rate of the system (from $\Omega \pm \Delta \Omega$ to $\Omega$ ). The flow stability depends on the characteristic values of the Rossby number, $\varepsilon=\Delta \Omega / \Omega$, and the Burger number, $B_{u}=N H / f R$, where $f=2 \Omega$ is the Coriolis parameter, $R$ is the characteristic horizontal length scale of the flow, $H$ is the depth of the fluid layer, and $N$ is the buoyancy frequency. Particular attention is given to the nonlinear flow regime (finite Rossby numbers). It is found that axisymmetric spin-up current loses its azimuthal symmetry when $B_{u}<1$, and breaks into a system of large-scale cyclonic and anticyclonic vortices with a predominantly vertical axis of rotation. The eddies always develop at the density fronts formed by the corner regions adjacent to the sidewalls of the container. The corner regions reach a quasi-equilibrium state at the characteristic time scale $E^{-1 / 2} \Omega^{1}$ (where $E=v / \Omega H^{2}$ is the Ekman number and $v$ is the kinematic viscosity), which is also observed for homogenous fluids. It is also shown that the stability of the spin-up flow is affected by the bottom slope. In the presence of the latter the bottom boundary layer experiences a qualitatively different behavior. While the density field demonstrates a smooth monotonic behavior in the case of stratified spin-up at all times, it reveals high-frequency fluctuations in the spin-down case, suggesting the turbulent nature of the bottom boundary layer. The results of observations may be found useful in interpreting in-situ measurements of upwelling- and downwelling-favorable oceanic currents in the littoral zones.
\end{abstract}

Keywords: geophysical systems, rotating stratified flows, spin-up, flow instability. 


\section{Introduction}

Dynamics of the spin-up flow initiated by a sudden acceleration/deceleration of the fluid container presents a fundamental interest from the point of view of the flow stability. Spin-up problem addresses an important question of how the torque exerted by the sidewalls of the fluid container is transmitted throughout the bulk of the rotating fluid. Although, the final condition of the fluid system is obvious - a new solid-body rotation state - it is far less clear what "routes" lead to this state. Spin-up in a homogeneous fluid has been extensively studied in the past (e.g., Greenspan [1], Duck and Foster [2]). It was shown that flow dynamics depends on the relative value of the Rossby number, $\varepsilon=\Delta \Omega / \Omega$. The flow evolution is governed by the Ekman layers formed on the top and bottom of the container. They initially convey the fluid towards the sidewalls into the Stewartson boundary layers. Passing through the boundary layers, fluid parcels roughly attain a new angular velocity of the container before moving into the interior. This secondary circulation is responsible for global spin-up of the fluid which is achieved at the homogeneous spin-up time scale $E^{-1 / 2} \Omega^{1}$.

In a stratified fluid gravity force limits the vertical fluid velocity and influences the secondary circulation. Flow dynamics is characterized by an additional parameter, the Burger number, $B_{u}=N H / f R$. The initial phase of stratified spin-up is similar to that of homogeneous spin-up (Benton and Clark [3], Buzyna and Veronis [4], Holton [5], Pedlosky [6]). Near-bottom heavy fluid is transported radially outward causing a strong deformation of isopycnals (surfaces of constant density) near the outer wall. This process is called upwelling. Stratified spin-up problem has been examined for different flow regimes in $\varepsilon-B_{u}$ parameter space, but the flow instability that breaks the axial symmetry was observed only for incremental spin-down (Hewitt et al $[7,8]$ ) or highly nonlinear spin-up from rest, when $\varepsilon=1$ (Flor et al [9, 10]). Nonaxisymmetric stage of incremental spin-up of a stratified fluid received special attention only recently (Kanda [11], Smirnov et al [12]). The formation of largescale eddies through the development of non-axisymmetric instabilities provides an alternative "route" in a stratified fluid for the transport of angular momentum from solid boundaries to the bulk of the fluid. This mechanism reduces the spinup time making it significantly less compared to the viscous time scale $E^{-1} \Omega^{1}$.

In the present paper we report on the cases of flow instability during incremental stratified spin-up and the conditions under which a sloping bottom topography may serve to stabilize the flow. Analysis of the boundary layer formed by an upwelling-favorable flow shows that buoyancy forces are always important unless $B_{\mathrm{u}}=0$ (MacCready and Rhines [13]). In the presence of a sloping bottom buoyancy inhibits the cross-slope transport. The density field redistribution in the horizontal plane due to the cross-slope transport establishes vertical shear through the thermal-wind balance. The decrease of the upslope transport prevents the heavier fluid from reaching the corner regions and closing the secondary meridional circulation. Buoyancy forces weaken the stress at the bottom boundary (free-slip boundary condition) and, therefore, affect the spin-up characteristics. 


\section{Experimental arrangements}

Laboratory experiments were conducted in a cylindrical tank (radius $R=91 \mathrm{~cm}$ ) positioned on a rotating table whose angular velocity was set within the frequency range $\left(0.1 \leq f \leq 1.0 \mathrm{~s}^{-1}\right)$ by a computer-controlled motor. The annular geometry was created by adding the second cylinder (radius $R_{1}=55 \mathrm{~cm}$ ) concentric with the outer cylinder. The bottom slope topography was modeled by an inverted cone (slope angle $\alpha=10^{\circ}$ ), which was centered inside a cylindrical container. The tank was stratified by salt using the standard two-bucket technique, so that the buoyancy frequency varied in the range, $0.5 \leq N \leq 2.0 \mathrm{~s}^{-1}$. The tank was covered with a transparent screen in order to reduce shear stress exerted by the air on the water free surface. The actual values of the stratification $(N)$ and rotation $(f)$ parameters were chosen to satisfy the condition $W f / N>H$, i.e. the height of a vertically sheared baroclinic current always exceeds the depth of the fluid layer at a given location.

Continuous stratification in the conical geometry was produced by two different methods. One method employed five fluid layers with uniform density increments. In this case the tank was stratified whilst at rest. The initial density profile always had a staircase shape, which was transformed into a linear one after three-four experimental runs. The second method employed a standard twobucket technique with a constant rate of filling, so that the tank can be stratified whilst rotating to reduce mixing. Measurements of the vertical density profiles were conducted prior to the beginning of spin-up in the center of the cone and at $r / R=0.54$ in order to estimate the effect of a sloping boundary on the background density profile (fig. 1). These two density profiles were almost identical from the free surface to the bottom of the cone at $r / R=0.54$. The density profile in the center of the cone preserved the same gradient over the next few centimeters and then started deviating sharply towards a constant-density profile (see almost flat curve in fig. 1) at the apex.

The flow was visualized using a neutrally buoyant dye-tracer (thymol blue $p \mathrm{H}$ indicator). Its evolution was recorded from above with a camera rotating with the tank. Particle tracking velocimetry (PTV) technique was employed to collect the quantitative data on the fluid velocity at different vertical levels. Small (mean diameter 100 microns) neutrally buoyant polystyrene particles were used as the flow tracers. They were distributed uniformly at various depths and illuminated with a light source from the side. The resulting motion was recorded from above and processed using DigImage software. The change in the rotation rate of the tank was performed during a five-second-time interval, $\Delta t$, and may be considered as impulsive $\left(\Delta t<<2 \pi \Omega^{-1}\right)$. The flow evolution was monitored for more than one hundred rotation periods.

Conductivity measurements were conducted using several four-electrode micro-scale conductivity probes. The data was collected using a computercontrolled acquisition system with a frequency of $10 \mathrm{~Hz}$. The voltage recordings were instantly reproduced in a graphical format and converted into salinity (density) values using the calibration curves. Finally, salinity values can be recalculated into the isopycnal displacements, $\eta$. 


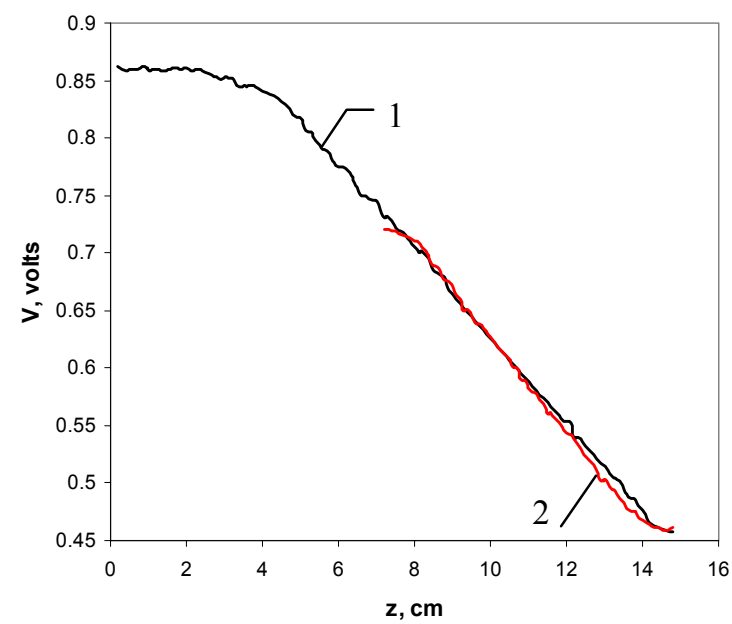

Figure 1: Dependence of the conductivity signal, $V$, on the vertical coordinate, $z$. Lines 1 and 2 represent the background vertical salinity profiles in the center of the cone $(r / R=0)$ and at $r / R=0.54$ respectively. The water free surface is at $z=15 \mathrm{~cm}$.

The accuracy of isopycnal displacement measurements was in the range \pm 0.1 $\mathrm{cm}$. The probes were located at various depths and radial positions from the center of the tank, which allowed one to investigate the vertical and horizontal density structure of the flow. Estimates of the wavelength of the instability (number of eddies in the system) were obtained by positioning two probes at the same radial distance from the center of the cylinder and separated by a known distance in the azimuthal direction. Knowing the frequency of each signal as well as the phase shift between them, one can estimate the propagation speed and the wavelength of the instability.

\section{Results}

A typical unstable flow pattern formed during stratified spin-up in the annulus with a flat bottom is shown in fig. 2 . The mean current is initiated in the clockwise direction. The dye tracer (dark bands) was introduced in the vicinity of both the outer and inner walls. The nature of the flow development in the annular region was found to be qualitatively similar to that in the cylinder. The dye streaks preserve their circular shape until $t / T \approx 30$. After about forty-five revolutions of the tank the flow becomes unstable as evidenced by the formation of eddies (fig. 2b). Cyclones and anticyclones originate independently near the outer and inner walls respectively advecting with the mean flow. Their growth is limited by the width of the annular channel. Two systems of eddies finally interconnect and form one "vortex street" (fig. 2c). Eventually, the system has spun up to the new solid-body rotation state and the flow pattern consisting of eddies of opposite signs remains visible for several hours (fig. 2d). 

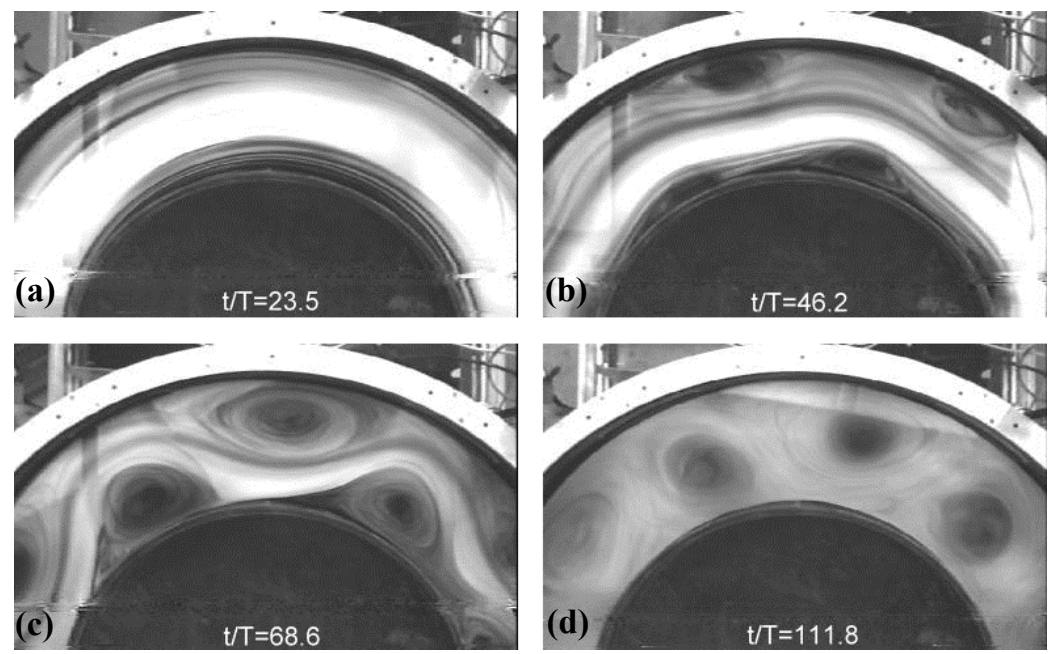

Figure 2: $\quad$ Stratified spin-up in the annulus. Top view. Parameters: $\varepsilon=0.12$, $B_{u}=1.08$. Dimensionless time: $t / T=$ (a) 23.5, (b) 46.2, (c) 68.6, and (d) 111.8 .

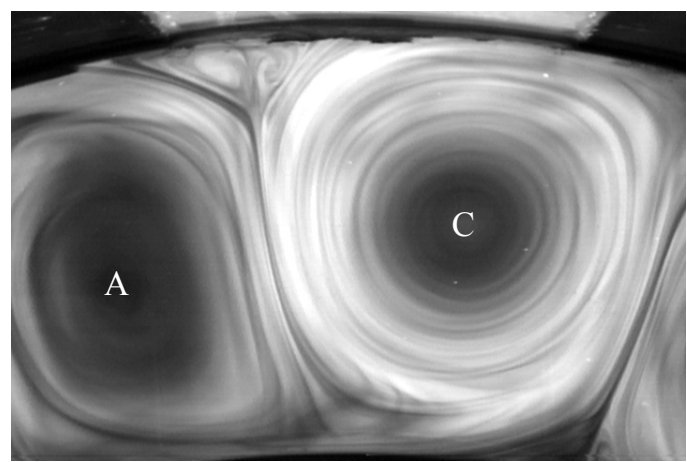

Figure 3: $\quad$ Magnified top-view image of the cyclonic (C) and anticyclonic (A) eddies formed during stratified spin-up in the annulus.

In fig. 3 a zoomed in image of the developed system of cyclones and aticyclones is shown with respect to the inner and outer sidewalls of the annulus which are located at the bottom and top parts of the image respectively. The direction of the spin-up current is again from left to right $\left(\varepsilon=0.167, B_{u}=0.58\right.$, $t / T=37.5$ ). Flow instability, when it occurs, is irreversible and has an explosive character. The growth of eddies continues until the whole flow field is occupied by a series of vortices with alternating signs. Their configuration sometimes has a peculiar form, like the one shown in fig. 3, where a large cyclone has a "tail" 
attached to the outer sidewall and composed of two smaller vortices of opposite signs. This "tail" slides along the sidewall without separation following the cyclone.

Non-axisymmetric instability is also clearly recognizable in PTV images. A typical example is shown in fig. 4.

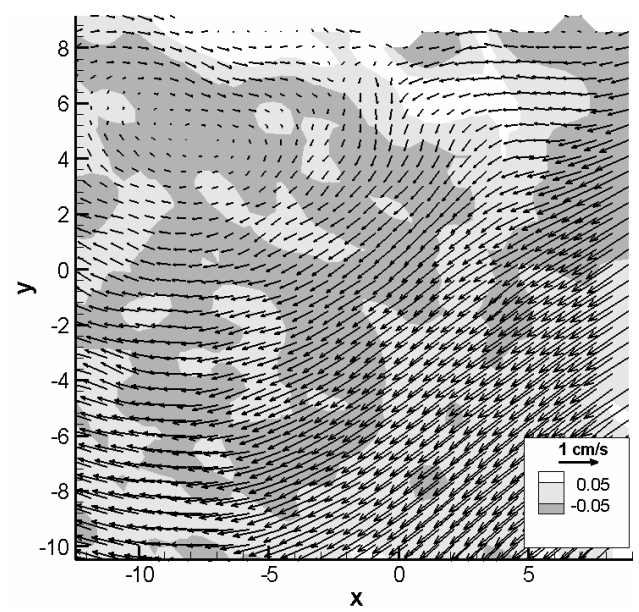

Figure 4: Velocity vector field and vorticity colour map representing stratified spin-up in the annulus. Top view. $X$ and $Y$ axes correspond to the azimuthal and radial directions respectively. The direction of the mean spin-up current is from right to left. Dark gray colour regions represent the negative (anticyclonic) vorticity. Parameters: $\varepsilon=0.107, B_{u}=0.55, t / T=48.1$.

The orientation of the velocity vectors changes throughout the image deviating significantly from the purely azimuthal direction. This explains the "patchiness" of the vorticity field.

Analysis of the density field evolution (not presented here) showed that eddies develop at the density front formed by the corner regions near the sidewalls. At this location the isopycnals experience the largest deformations as a result of the Ekman meridional transport. The latter makes the corner regions grow during the transient time $E^{-1 / 2} \Omega^{-1}$, if $B_{u}<1$, or $B_{u}{ }^{-1} T_{\mathrm{S}}$, if $B_{u}>1$, until they reach a quasi-equilibrium height determined by the relative values of $\varepsilon$ and $B_{u}$. The density structure of the corner regions was found to be the key factor in determining the stability of stratified spin-up flows.

The evolution of the isopycnal displacements at a given location is shown in fig. 5 for the annular geometry. The tracking of the conductivity signal was conducted at three different depths and at the same distance form the center of rotation. Because all probes were located approximately in the middle of the channel, there is no pronounced increase or decrease of the density at early times. Undulations of the signals correspond to the developing instability. The 
instability develops in the form of eddies, which are represented by regular lowfrequency (about $0.1 f$ ) oscillations in fig. 5. Note that oscillations at different levels are practically in phase and the largest isopycnal deformation is registered closer to the bottom of the annulus, while its manifestation close to the free surface is relatively weak.

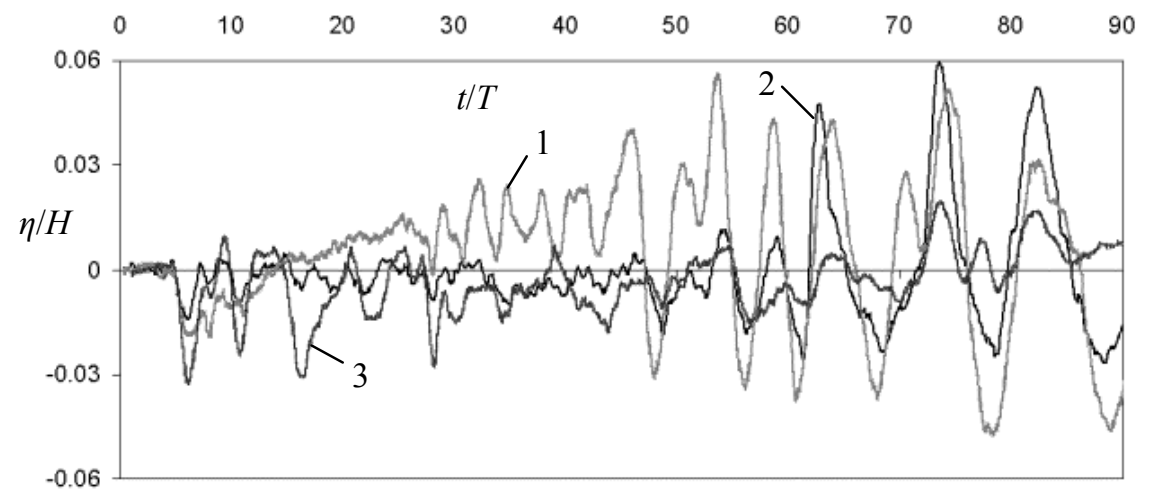

Figure 5: Time evolution of the normalized isopycnal displacements during stratified spin-up in the annulus. Conductivity probes are distributed vertically at $z=$ (1) 2.5 , (2) 6 , and (3) $10 \mathrm{~cm}$ from the bottom of the annulus $(H=12.5 \mathrm{~cm}$ is the total depth of the fluid layer) and positioned at $r=74 \mathrm{~cm}$ from the center of rotation. Time $t=0$ represents the beginning of measurements, while the end of the flat region corresponds to the initiation of spin-up. Parameters: $\varepsilon=0.12, B_{u}=0.7$.

Stratified spin-up/down in a conical geometry appeared to be fundamentally different from the above cases. The flow evolution visualized with a passive dye tracer is shown in fig. $6 \mathrm{a}$ (stable regime) and $6 \mathrm{~b}$ (unstable regime). The absolute values of the flow regime parameters, $\varepsilon$ and $B_{\mathrm{u}}$, were kept the same in both cases. The passive dye tracer, introduced near the bottom, follows the boundary-layer current. The difference in the flow patterns becomes clear after a few rotation periods. A system of relatively small and densely packed anticyclonic eddies spawns out of the initially chaotic distribution of the dye tracer. These eddies merge and finally form a system of much larger anticyclonic vortices (fig. 6b). Contrary to that, in the spin-up case the dye tracer dispersed by the shear flow preserves its axisymmetry even at very late times of the flow evolution (fig. 6a). The observations suggest that stratified spin-up flows remain stable at all times.

A comparison of the vertical density profiles for positive (spin-up) and negative (spin-down) Rossby numbers shows that in the former case the density gradient decreases (compared to the background value) in the upper part of the fluid column and increases near the bottom, while in the latter case this tendency is reversed. This observation indicates to the existence of intensive mixing regions localized in the vicinity of the bottom boundary in the spin-down case. 

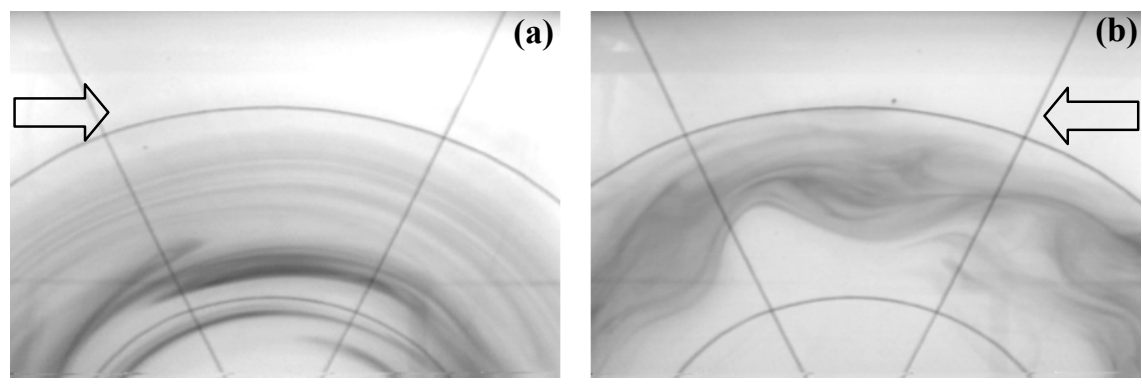

Figure 6: $\quad$ Stratified spin-up/down in the axisymmetric conical geometry. Top view. The block arrow shows the direction of the mean current. The radial distance between two concentric rings is $30 \mathrm{~cm}$. Parameters: (a) spin-up $\varepsilon=0.3, B_{u}=0.42, t / T=41.5$, (b) spin-down $\varepsilon=-0.3, B_{u}=0.42, t / T=22.6$.

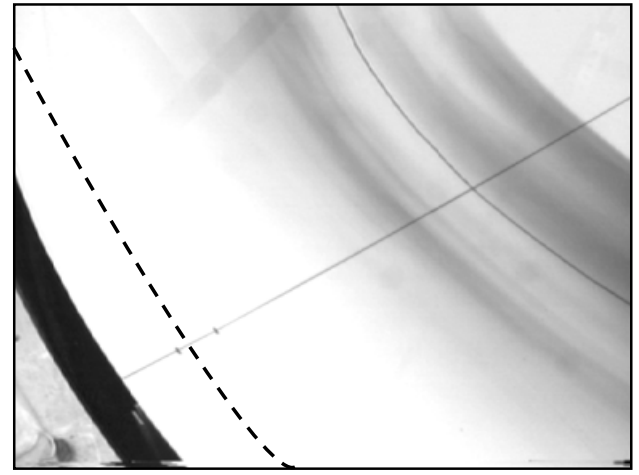

Figure 7: Dye visualization of stratified spin-up in the shallow part of the conical geometry. Parameters: $\varepsilon=0.3, B_{u}=0.46, t / T=102$. The distance between two marks along the radius is $3 \mathrm{~cm}$. The dark band in the left bottom corner corresponds to the outer edge of the cone. The free surface intersects the sloping bottom at the location shown by the broken line.

The differences between spin-up and spin-down flow patterns may also be delineated through measurements of instantaneous vertical salinity profiles. It was found that the fundamental difference between stratified spin-up and spindown is manifested in the way the boundary layer behaves shortly after the change in the rotation rate of the container. In the case of spin-up the conductivity probe positioned inside the boundary layer registered a smooth and monotonic increase in density, while in the case of spin-down the registered signal experienced a large number of random high-frequency oscillations shortly after the beginning of spin-down. This observation suggests that the 
downwelling boundary-layer current becomes unstable and turbulent relatively fast, while upwelling boundary-layer remains stable at all times. This, in turn, leads to qualitatively different boundary conditions for the interior flow.

The segment of the conical geometry shown in fig. 6 represents the mid-depth region of the flow domain, with the lower and upper circles being at $30 \mathrm{~cm}$ and $60 \mathrm{~cm}$ respectively from the center of the cone measured along the bottom of the tank. In general, the flow evolution is expected to be different in the deep and shallow parts of the flow domain and, therefore, the flow region where the sloping bottom intersects the free surface needs special attention as a potential source of flow instability. Experiments conducted with the dye tracer being introduced in the vicinity of the outer edge of the tank (fig. 7) showed that even after one hundred revolutions the stratified spin-up flow remains axisymmetric in this part of the conical geometry, which means that it is globally stable. It is also interesting to note, that in the case of spin-down the eddies do not form close to the outer rim of the cone, where the fluid reaches a new solid-body rotation relatively quickly because of the small depth of the fluid layer, but rather in the mid-depth section of the tank shown in fig. 6 .

\section{Conclusions}

It was demonstrated that spin-up flows of rotating, continuously stratified fluid in axisymmetric geometries may become unstable at late times (several tens of rotation periods). The instability and formation of cyclonic/anticyclonic eddies may be suppressed by increasing $B_{u}$ and decreasing $\varepsilon$. The eddy formation time decreases for larger $\varepsilon$ and is independent of $B_{u}$ (for the range of parameters investigated). Unquestionably the bottom topography plays a crucial role in setting up the density stratification and vertical shear in the bottom boundary layer that influences both the development of non-axisymmetric instabilities and global spin-up. If the characteristic "shutdown" time is relatively small, the heavier fluid may never reach the corner regions and the formation of the "corner jets", which close the meridional circulation, does not occur. Thus, the slippery boundary condition should affect the global spin-up time of the interior fluid.

\section{Acknowledgements}

The author is grateful to Professors H.J.S. Fernando and D.L. Boyer for the support of this research under ONR grant N00014-0-1-0626 and NSF grant OCE-0137197.

\section{References}

[1] Greenspan, H.P., The Theory of Rotating Fluids, Cambridge Univ. Press: London and New York, 1968.

[2] Duck, P.W. \& Foster, M.R., Spin-up of homogeneous and stratified fluids. Ann. Rev. Fluid Mech., 33, pp. 231, 2001. 
[3] Benton, E.R. \& Clark, A., Spin-up. Ann. Rev. Fluid Mech., 6, pp. 257, 1974.

[4] Buzyna, G. \& Veronis, G., Spin-up of a stratified fluid: theory and experiment. J. Fluid Mech., 50, pp. 579, 1971.

[5] Holton, J.R., The influence of viscous boundary layers on transient motions in a stratified rotating fluid: Part I. J. Atmos. Sci., 22, pp. 402, 1965.

[6] Pedlosky, J., The spin up of a stratified fluid. J. Fluid Mech., 28, pp. 463, 1967.

[7] Hewitt, R.E., Davies, P.A., Duck, P.W. \& Foster, M.R., Spin-up of stratified rotating flows at large Schmidt number: experiment and theory. J. Fluid Mech., 389, pp. 169, 1999.

[8] Hewitt, R.E., Foster, M.R. \& Davies, P.A., Spin-up of a two-layer rotating stratified fluid in a variable-depth container. J. Fluid Mech., 438, pp. 379, 2001.

[9] Flor, J.B., Ungarish, M. \& Bush, J.W.M., Spin-up from rest in a stratified fluid: boundary flows. J. Fluid Mech., 472, pp. 51, 2002.

[10] Flor, J.B., Bush, J.W.M. \& Ungarish, M., An experimental investigation of spin-up from rest of a stratified fluid. Geophys. Astrophys. Fluid Dyn., 98, pp. 277, 2004.

[11] Kanda, I., A laboratory study of columnar baroclinic vortices in a continuously stratified fluid. Dyn. Atmos. Oceans, 38, pp. 69, 2004.

[12] Smirnov, S.A., Baines, P.G., Boyer, D.L., Voropayev, S.I. \& SrdicMitrovic, A.N., Long-time evolution of linearly stratified spin-up flows in axisymmetric geometries. Phys. Fluids, 17, 016601, 2005.

[13] MacCready, P. \& Rhines, P., Buoyant inhibition of Ekman transport on a slope and its effect on stratified spin-up. J. Fluid Mech., 223, pp. 631, 1991. 